to teach were adults who were training for qualification in her specialty. In contrast, doctors are often required to teach members of the health care professions despite having had no instruction in teaching skills and practice.

\section{Gaining teaching skills}

How may doctors gain the skills necessary to teach medicine to others? Clearly the investment of time entailed in training full time teachers is both unnecessary and out of the question, but what about a basic training course in teaching skills and practice? In practical terms this would probably have to be in doctors' own time because of the heavy workloads and probably at their own expense because of financial restrictions in the NHS. A weekend conference may well be most suitable, but if health authority approval could be obtained a period of study leave could be arranged. The course should comprise lectures and practical experience, the latter arranged by requiring each participant to present a lecture to the rest of the group, with subsequent advice and critique.

And how should the course be followed up? Continuing self evaluation using written critiques from all the people who are subsequently taught could be considered based on a format provided during the training course. Though it is a sensitive suggestion, other NHS staff might be invited to give regular critiques on the doctor's performance at work. For those doctors offended by such a suggestion I report that my wife's teacher friend occasionally has to sit in front of her adult class, with her fellow instructors present, while the students deliver their critiques. Anyway, the following advice may help doctors to be better teachers.

- Bearing: be bold and confident, stand upright, and avoid distracting mannerisms

- Be well prepared: adopt objectives for each lesson so that nothing is missed out and the lesson follows a logical progression; teaching off the cuff is not as effective

- Use a variety of teaching methods: this is essential to retain the students' interest-useful ideas include scenarios, interesting slides or a film, question and answer sessions, anecdotes or examples from your own experience, group discussions, good handouts, etc

- Vary your tone of voice: a monotone induces sleep

- Pitch the lecture at the right level: find out beforehand the students' abilities and levels of experience.

In conclusion doctors who have to teach cannot be assumed to possess natural teaching skills. Instruction in basic teaching skills could usefully be provided. Judged by the recent student critiques I am now considered a good teacher. Meanwhile my respect for full time teachers has increased considerably: I had no idea there was so much to teaching.

\title{
Medicine and the Law
}

\section{How informed is signed consent?}

\author{
D J BYRNE, A NAPIER, A CUSCHIERI
}

\begin{abstract}
The assurance that patients fully understand the information given to them before they sign the consent form for operation has never been validated in this country. One hundred patients were interviewed by an independent medical observer in one surgical unit in a teaching hospital between two and five days after their operation. Although all the patients interviewed were fully aware that they had had an operation, 27 did not know which organ was operated on and 44 were unaware of the exact nature of the surgical procedure. A significantly higher age group was observed in the group of patients who were unaware of the basic facts relating to their operation, and the problem was encountered most commonly in patients over the age of 60 . Because of the
\end{abstract}

\footnotetext{
Department of Surgery, Ninewells Hospital and Medical School, University of Dundee, Dundee DD1 9SY

D J BYRNE, MB, FRCSI,

A NAPIER, RGN, nurse

A CUSCHIERI, MD, FRCS, professor and head of department of surgery

Correspondence to: Professor A Cuschieri, Department of Surgery, Queen Elizabeth Hospital, Edgbaston, Birmingham B15 2TH
}

medical and legal importance the findings of this pilot study warrant further large scale investigations.

\section{Introduction}

Informed signed consent is now a legal necessity for all surgical procedures. ${ }^{1}$ In this country it is increasingly considered desirable, if not mandatory, before recruiting patients into clinical trials..$^{23}$ In the United States such signed informed consent is a legal requirement. ${ }^{4}$ But in this country the reliability of medical communication and assurance that patients fully understand the medical terminology and information given to them has been assumed but never validated. Studies in the United States have found poor understanding and recall of consent form information. ${ }^{5.7}$ Most of the patients in these trials, however, were undergoing various treatments other than surgery, the information they were asked to recall was rather detailed-for example, the surgical techniques to be employed-and their recall was tested up to two years after signing the consent form. In addition to the communication skills and experience of the medical attendant, several factors may adversely influence the ability of the patient to understand, appreciate, and retain the information.

In this preliminary study we tried to ascertain the level of knowledge of 100 consecutive patients treated in one surgical unit about the nature of the operation performed before they were discharged. 


\section{Patients and methods}

The study was done in a 48 bed academic surgical unit run by a professor and two senior lecturers. The ward procedure was not changed for the study. There is a consultant round the day before the operation when the consultant tells the patient of the nature of the intended operation. Subsequently, on the same day, the patient is interviewed by a junior member of staff-house surgeon or senior house officer-who repeats the details of the intended surgical treatment and obtains the signed consent from the patient on a standard hospital surgical consent form.

In this study an independent surgically qualified observer or a registered general nurse with no clinical commitments to the surgical unit (DB, AN) interviewed the patients between the second and fifth days after their operations requesting information on the following: (i) awareness of having had an operation; (ii) knowledge of the organ operated on; and (iii) basic details of the operation performed.

One hundred patients were included in the study -59 women and 41 men-within an age range of 16 to 84 years (mean 55 years). None of the patients in the study was psychologically disturbed or showed evidence of disorientation although we did not perform a specific audit of the emotional, psychological, and mental state of the patients.

\section{Results}

Table I gives the details of the various operations performed. As the responses from patients obtained by the two interviewers (DB, AN) were similar and were not statistically significant $\left(\chi^{2}=0 \cdot 137\right)$ the data collected by the two observers were pooled.

\begin{tabular}{lc}
$\begin{array}{l}\text { TABLE I-Type and number of } \\
\text { performed }\end{array}$ & No \\
\hline Procedure & 38 \\
\hline Cholecystectomy & 10 \\
Aortoiliac bypass procedures & 10 \\
Colectomy & 8 \\
Antireflux procedures (Niessen, Belsy) & 5 \\
Gastrectomy & 4 \\
Vagotomy and drainage & 3 \\
Highly selective vagotomy & 4 \\
Small bowel resection & 18 \\
Other procedures & 100 \\
\hline \multicolumn{2}{c}{ Total }
\end{tabular}

TABLE $\mathrm{II}-K$ nowledge of operation in relation to age of patients

\begin{tabular}{lcc}
\hline \multirow{2}{*}{ Information } & \multicolumn{2}{c}{ Age (mean SD)) } \\
\cline { 2 - 3 } & Aware & Not aware \\
\hline Organ operated on & $51 \cdot 5(15 \cdot 3)$ & $64 \cdot 4(8 \cdot 6)^{\star}$ \\
Procedure on organ & $49 \cdot 6(15 \cdot 6)$ & $61 \cdot 9(10 \cdot 6)^{\star}$ \\
\hline${ }^{*} \mathrm{p}<0.001$. & & \\
\end{tabular}

On the day of the interview (second to fifth day after operation) all the patients were alert, fully conscious, and not sedated. All were aware that they had undergone and were recovering from a surgical procedure, but 27 did not know which organ was operated on and 44 were unaware of the basic facts relating to the operation-for example, that the gall bladder had been removed. Technical information about the procedure carried out was not requested by the interviewer. When the replies were assessed in relation to age the elderly patients lacked the basic information relating to their recent operation more often than did the patients aged 50 and under (table II).

\section{Discussion}

This survey has shown that although the signed consent form before surgical treatment fulfills a legal requirement, it in no way guarantees that the patient is fully aware of the exact nature of the treatment. The problem seems to be particularly prevalent in the elderly although it is not confined to this age group.

There are several possible explanations for the unsatisfactory state of affairs. The information given by the surgical staff of the unit was possibly inadequate because of insufficient time, because the medical terminology was poorly understood by the patients, and because of a lack of basic communication skills on the part of the medical attendants. Although this cannot be refuted, it seems unlikely to be the major factor. But it is an aspect that requires further investigation.

The high incidence of the problem in the elderly suggests that cerebral atrophy, with loss of memory for recent events, aggravated by a general anaesthetic and the administration of sedative analgesic drugs, was probably important in this age group. If this is substantiated by subsequent reports then the procedure for obtaining consent for operation in the elderly may have to be revised; the presence of a younger relative at the consent signing may be necessary.

It would be rash to make firm recommendations on the results of this small pilot study. But it has shown that larger surveys should consider other factors known to influence patients' understanding of medical information. In a previous report the main factors responsible for inadequate recall of consent form information were level of education, medical state, and the care with which the patient reads the consent form before signing. ${ }^{7}$

Finally, there seems to be a group of patients who are distressed by the "gory details" of an operation and who simply want to be treated surgically or otherwise so that they can leave the hospital, forget their illness and, resume growing prize marrows as far away from the medical confraternity as possible.

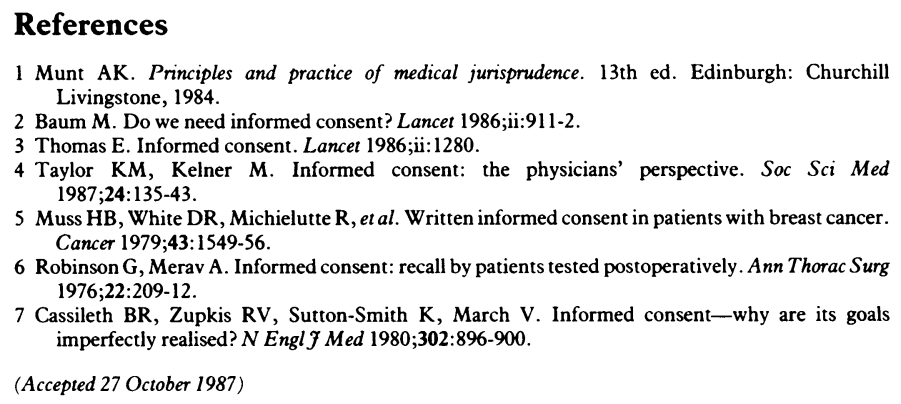

Is a history of epilepsy in first degree relatives a contraindication to pertussis and measles vaccination?

No. A history of epilepsy in a first degree relative (parent, brother, or sister) is not an absolute contraindication to these immunisation procedures, but immunisation does require special consideration. For pertussis the doctor should assess each case individually, taking into account whether any febrile episodes in the past have caused convulsions and attempting to weigh the relative risks of one or more seizures following, or potentially provoked by, either the vaccine or the disease itself. Measles can provoke convulsions, which may also more rarely occur after administration of the vaccine. In the hope of reducing reaction to measles vaccine the Joint Committee on Vaccination and Immunisation now recommends that children with a personal history of convulsions or whose parents or siblings have a history of idiopathic epilepsy should receive an injection of human normal immunoglobulin for use with measles vaccine but given at a different site This is a specially diluted preparation intended to minimise reaction to measles vaccine without neutralising it, and is available from the Blood Products Laboratory, Elstree, or in Scotland from the blood transfusion service.-N R GRIST, emeritus professor of infectious diseases, Glasgow. 